\title{
Compound Fault Diagnosis of Bearings Using an Improved Spectral Kurtosis by MCDK
}

\author{
Shuting Wan, Xiong Zhang $(\mathbb{D}$, and Longjiang Dou $(\mathbb{D}$ \\ Department of Mechanical Engineering, North China Electric Power University, Baoding 071003, China \\ Correspondence should be addressed to Xiong Zhang; hdjxzx@ncepu.edu.cn
}

Received 8 December 2017; Revised 8 January 2018; Accepted 7 February 2018; Published 19 March 2018

Academic Editor: Aimé Lay-Ekuakille

Copyright (C) 2018 Shuting Wan et al. This is an open access article distributed under the Creative Commons Attribution License, which permits unrestricted use, distribution, and reproduction in any medium, provided the original work is properly cited.

\begin{abstract}
The fast spectrum kurtosis (FSK) algorithm can adaptively identify and select the resonant frequency band and extract the fault feature by the envelope demodulation method. However, in practical applications, the fault source may be located in different resonant frequency bands; plus in noise interference, the weak side of the compound fault is not easy to be identified by the FSK. In order to improve the accuracy of fast spectral kurtosis analysis method, a modified method based on maximum correlation kurtosis deconvolution (MCKD) is proposed. According to the possible fault characteristic frequencies, the period of MCKD is calculated, and the appropriate filter length is selected to filter the original compound fault signal. In this way, the compound fault located in different resonance bands is separated. Then, the signal after MCKD filtering is analyzed by FSK. Through the simulation and experimental analysis, the MCKD can separate the compound fault information in different frequency band and eliminate the noise interference; the FSK can accurately identify the resonance frequency and identify the weak fault characteristics of compound fault.
\end{abstract}

\section{Introduction}

The fault characteristics of rotating machinery have certain regularity and periodicity. The application of vibration signal and acoustic signal to the fault feature extraction of rotating machinery fault is of great value [1]. Rolling bearing is one of the most widely used parts in mechanical equipment. Its operation condition affects the working state of the whole system [2-5]. The fault identification and diagnosis of rolling bearing are of great significance to ensure the safe and reliable operation of mechanical equipment. Under the actual conditions, the failure of rolling bearing usually manifests itself as compound failure, and due to the influence of operating environment, the interaction between multiple noise source and compound fault is often presented. The separation of compound fault components from strong background noise is a difficult problem in the field of mechanical fault diagnosis [6-8]. Hemmati et al. proposed a modified and effective signal processing algorithm to diagnose localized defects on rolling element bearings components under different operating speeds, loadings, and defect sizes [9]. The algorithm was based on optimizing the ratio of kurtosis and Shannon entropy to obtain the optimal band pass filter utilizing wavelet packet transform and envelope detection. The performance of ELMD often heavily depends on proper selection of its model parameters; to overcome this shortcoming, Zhang et al. propose an optimized ensemble local mean decomposition method to determinate an optimum set of ELMD parameters for vibration signal analysis [10].

The concept of spectrum kurtosis is presented by Dwyer. The basic principle is to calculate the kurtosis value of each spectral line, and the value of different kurtosis will respond to the transient impact size. Antoni and Randall used the spectral kurtosis value as a short time Fourier window function and selected the parameters of the bandpass filter through the spectral kurtosis diagram and proposed the spectral kurtosis discrete algorithm [11]. For improving the kurtosis figure in the engineering application of operational efficiency and real-time, Antoni and Randall propose a fast kurtograph algorithm based on binary finite impulse response filter group. The wavelet kurtograph algorithm is proposed based on the nonorthogonal complex wavelet analysis 
by Sawalhi et al. [12], and the frequency band partition in the rapid spectrum is optimized. Liu et al. propose an adaptive spectral kurtosis filtering technique to extract the signal transients based on Morlet wavelet. The Morlet wavelet is used as a filter bank whose center frequency is defined by the wavelet correlation filtering [13].

The maximum correlation kurtosis deconvolution (MCKD) is proposed by McDonald et al. [14]. It is a method to extract weak impact components by raising the kurtosis of signals to achieve the purpose of noise reduction under low signal-to-noise ratio. Compared with the minimum entropy deconvolution (MED), MCKD has obvious advantages in dealing with strong background noise. When a fault occurs, we can define the discrete signal $x(n)$ as the response of the bearing excited by the faulty impulse signal $y(n)$. MCKD searches for a FIR filter $f$ to maximize the correlation kurtosis of the signal $y(n)$ recovered from the input signal and the general expression for the inverse is given by

$$
y(n)=\sum_{k=1}^{L} f(k) x(n-k+1),
$$

where $l=1,2, \Lambda, L$, and $L$ is the length of the FIR filter.

The correlation kurtosis is defined as

$$
\mathrm{CK}_{M}(T)=\frac{(1 / n) \sum_{n=1}^{N}\left(y_{n} y_{n-T}\right)^{2}}{\left((1 / n) \sum_{n=1}^{N} y_{n}^{2}\right)^{2}}-3,
$$

where $N$ represents the length of the signal, $T$ is the deconvolution cycle, and $M$ is the number of conversions.

The optimization function of MCKD is defined as

$$
\max _{f} \mathrm{CK}_{M}(T)=\max _{f} \frac{\sum_{n=1}^{N}\left(y_{n} y_{n-T}\right)^{2}}{\left(\sum_{n=1}^{N} y_{n}^{2}\right)^{2}} .
$$

The optimization function is to find the optimal filter which maximizes the correlation kurtosis $\mathrm{CK}_{M}(T)$. The calculation formula is defined as

$$
\frac{d}{d f_{k}} \mathrm{CK}_{M}(T)=0, \quad(k=1,2, \ldots, L) .
$$

The FIR filter result of $f$ is defined as

$$
f=\frac{\|y\|^{2}}{2\|\beta\|^{2}}\left(\vec{X}_{0} \vec{X}_{0}^{T}\right)^{-1} \sum_{m-0}^{M} \vec{X}_{m T} \alpha_{m},
$$

where

$$
\begin{array}{r}
\vec{X}_{r}=\left[\begin{array}{cccc}
x_{1-r} & x_{2-r} & \cdots & x_{N-r} \\
0 & x_{1-r} & \cdots & x_{N-1-r} \\
\vdots & \vdots & \vdots & \vdots \\
0 & 0 & \cdots & x_{N-L-r+1}
\end{array}\right]_{L \times N} \\
\\
(r=0, T, 2 T, \ldots, M T)
\end{array}
$$

$$
\begin{gathered}
\alpha_{m}=\left[\begin{array}{c}
y_{1-m T}^{-1}\left(y_{1}^{2} y_{1-T}^{2} \cdots y_{1-M T}^{2}\right) \\
y_{2-m T}^{-1}\left(y_{2}^{2} y_{2-T}^{2} \cdots y_{2-M T}^{2}\right) \\
\vdots \\
y_{N-m T}^{-1}\left(y_{N}^{2} y_{N-T}^{2} \cdots y_{N-M T}^{2}\right)
\end{array}\right]_{N \times 1}, \\
\beta=\left[\begin{array}{c}
y_{1} y_{1-T} \cdots y_{1-M T} \\
y_{2} y_{2-T} \cdots y_{2-M T} \\
\vdots \\
y_{N} y_{N-T} \cdots y_{N-M T}
\end{array}\right]_{N \times 1} .
\end{gathered}
$$

Fast spectral kurtosis is sensitive to noise, and it is easy to cause misdiagnosis or missed diagnosis for compound fault in different resonance frequency bands.

When determining the resonance band, the FSK can only select the resonance band with the maximum kurtosis. The information of compound fault is often distributed in two different resonance bands. Only when the kurtosis values of the two resonance bands are very close to each other, the FSK can identify two resonance bands accurately. However, for most cases, the compound fault has a large difference in the kurtosis values of the two resonance band components. This results in omission of part of the fault information when dealing with compound failure problems with FSK. Moreover, when the noise is relatively large, in the interference of noise, the two resonance bands may lose. The simulation signal with the impact characteristics located at two different resonance bands is defined as

$$
\begin{aligned}
y= & A_{1} e^{-\xi\left[t-q_{1}(t) / f_{1}\right]^{2}} \cdot \sin \left(2 \pi f_{n 1} t\right)+A_{2} e^{-\xi\left[t-q_{2}(t) / f_{2}\right]^{2}} \\
& \cdot \sin \left(2 \pi f_{n 2} t\right)+n(t),
\end{aligned}
$$

where $f_{n 1}=1500 \mathrm{~Hz}$ is the first resonance frequency, $f_{n 2}=$ $3000 \mathrm{~Hz}$ is the second resonance frequency, $f_{1}=50 \mathrm{~Hz}$ and $f_{2}$ $=125 \mathrm{~Hz}$ are two characteristic frequencies, $\xi$ is the damping ratio, $A_{1}$ and $A_{2}$ are the amplitudes, $q_{d}(t)=\left\lfloor t \cdot f_{d}\right\rfloor(d=1,2)$, and $n(t)$ is the Gauss white noise. The problems encountered by FSK in analyzing compound fault are verified by adjusting the numerical values of $A_{1}, A_{2}$, and $n(t)$.

Figure 1(a) shows the kurtosis values of the two impact characteristics are similar in magnitude and the noise interference is small. The impact information located in different resonance bands can be extracted by the FSK method. Filtering is carried out in the two resonant frequency bands, and then the envelope spectrum analysis is performed to extract the characteristic frequencies of the two impact characteristics. Then the proportion of two impacts is changed by changing the size of $\mathrm{A} 1$ and A2. Figure 1(b) shows that the resonance band information of a relatively weak impact characteristic is submerged because of the changing proportion of the two impacts. Figure 1(c) shows that the two resonance bands are submerged when the noise is large. The above analysis shows that noise and compound fault may 

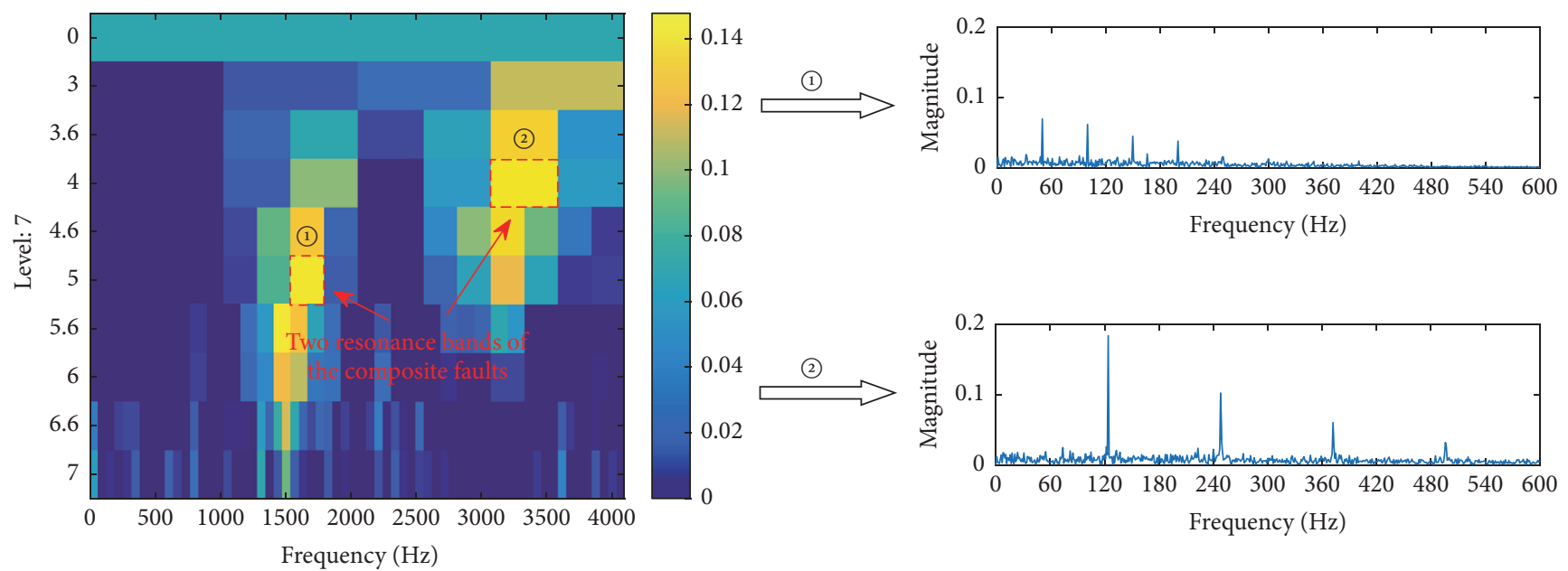

(a) Two resonance bands and their envelope spectrum identified by FSK

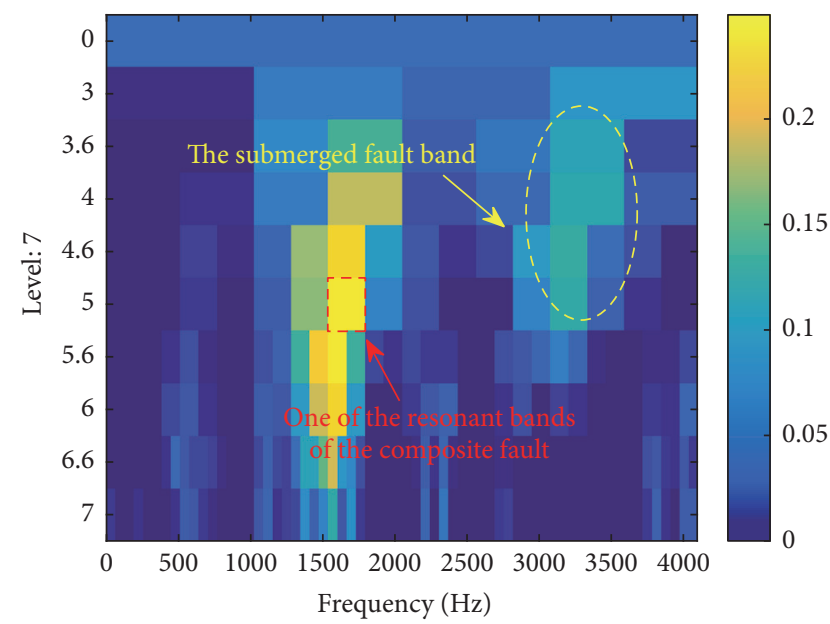

(b) The limitation of FSK to compound fault identification

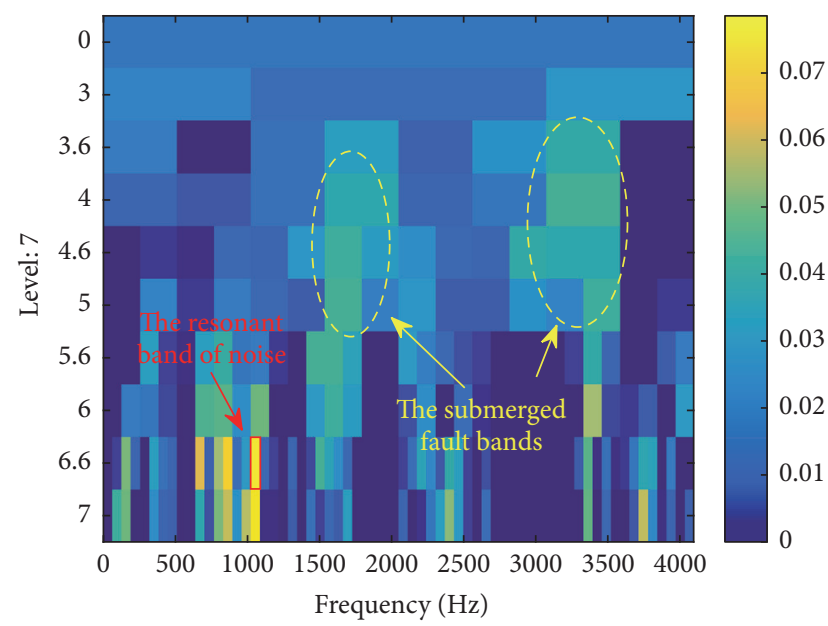

(c) The limitations of FSK in identifying compound fault under noise interference

FIGURE 1: Selection of resonance band of compound fault by FSK.

cause inaccuracy when determining the resonant bands by FSK.

\section{The Improved FSK with MCKD}

FSK has poor performance in fault feature extraction in different resonance frequency bands. In order to overcome this shortcoming, MCKD is used as preprocessing. The flow chart of the fault feature extraction of the bearing is shown in Figure 2. The specific process is as follows:

(1) The theoretical characteristic frequency of bearing fault is calculated, according to the parameter of bearing. The empirical formula of fault characteristic frequency is defined as

$$
\begin{aligned}
& f_{i}=\frac{Z}{2}\left(1+\frac{d}{D} \cos \alpha\right) \frac{N}{60}, \\
& f_{\mathrm{o}}=\frac{Z}{2}\left(1-\frac{d}{D} \cos \alpha\right) \frac{N}{60},
\end{aligned}
$$

$$
\begin{aligned}
& f_{\text {ball }}=\frac{D}{2 d}\left(1-\left(\frac{d}{D}\right)^{2} \cos ^{2} \alpha\right) \frac{N}{60}, \\
& f_{\text {cage }}=\frac{1}{2}\left(1-\frac{d}{D} \cos \alpha\right) \frac{N}{60},
\end{aligned}
$$

where $f_{i}, f_{0}, f_{\text {ball }}$, and $f_{\text {cage }}$, respectively, represent the inner ring fault, outer ring fault, rolling body element fault, and cage fault. $Z$ is the number of ball elements. $d$ is the diameter of the rolling element. $D$ is the pitch diameter. $N$ is the spindle speed. $\alpha$ is the pressure angle.

(2) The deconvolution period is obtained from the theoretical fault characteristic frequency mentioned above. The empirical formula of deconvolution period is defined as

$$
T_{*}=\frac{f_{s}}{f_{*}} \quad(* \text { is } i, o \text {, ball or cage }),
$$






Figure 2

where $T_{*}$ is the period of MCKD. $f_{s}$ is the sampling frequency. $f_{*}$ is the characteristic frequency of fault calculated by theoretical calculation.

(3) Determine the filter length of the MCKD. In the process, the maximum correlation kurtosis is used as the parameter to optimize the target, with 10 as the step length and 5 to 200 as the search range. Finally, $L_{*}$ is determined to be the optimum filter length.

(4) The original signal is filtered by the combination of the MCKD parameters $\left[T_{*}, L_{*}\right]$. After filtering, several sets of time domain signals with different fault are obtained. That is, the impact information located in the different resonance bands is separated.

(5) The signals obtained in step (3) are analyzed by FSK, respectively, and the resonance frequency bands of each signal are determined. The resonant frequency bands are filtered, respectively, and the filtered signals are analyzed by envelope spectrum. The characteristic spectrum of the fault is obtained from the envelope spectrum.

\section{Simulation Analysis}

The compound fault signal is simulated by the simulation signal of the inner ring and outer ring. The inner ring fault signal simulation model is defined as in the following equation [15].

$$
\begin{aligned}
x(t) & =s(t)+n(t)=\sum_{i} A_{i} h\left(t-i T-\tau_{i}\right)+n(t) \\
A_{i} & =A_{0} \cos \left(2 \pi f_{r} t+\phi_{A}\right)+C_{A} \\
h(t) & =e^{-B t} \cos \left(2 \pi f_{n} t+\phi_{\omega}\right),
\end{aligned}
$$

where $\tau_{i}$ is the minor fluctuation of the $i$ shock relative to the period $T ; A_{i}$ is the amplitude modulation with $1 / f_{r}$ as a cycle; $h(t)$ is an exponential decay pulse; $B$ is the attenuation coefficient of the system; $\phi_{A}, \phi_{\omega}$ are the initial phase. The parameters of the inner ring simulation signal are shown in Table 1.

The outer ring fault simulation signal is defined as

$$
x=y_{0} e^{\left(-2 g \pi f_{n} t_{0}\right)} \cdot \sin \left(2 \pi f_{n} \sqrt{1-g^{2}} \cdot t_{0}\right),
$$


TABLE 1: The parameters of inner fault signal simulation.

\begin{tabular}{lccccc}
\hline Amplitude & Rotating frequency & Natural frequency/Hz & Sampling frequency/Hz & Sampling points/Hz & Fault frequency/Hz \\
$A_{0} / \mathrm{Hz}$ & 3600 & 8192 & 8192 \\
\hline 1 & 20 & 130 & \\
\hline
\end{tabular}

TABLE 2: The parameters of the outer ring fault simulation signal.

\begin{tabular}{lccccc}
\hline $\begin{array}{l}\text { Amplitude } \\
A_{0}\end{array}$ & $\begin{array}{c}\text { Rotating frequency } \\
f_{r} / \mathrm{Hz}\end{array}$ & Natural frequency/Hz & Sampling frequency/Hz & Sampling points/Hz & Fault frequency/Hz \\
\hline 2 & 20 & 1600 & 8192 & 8192 & 50 \\
\hline
\end{tabular}

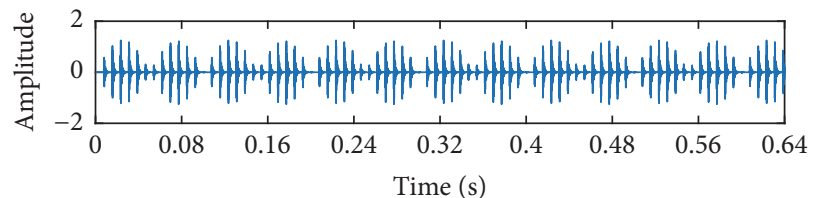

(a) Inner ring fault simulation signal

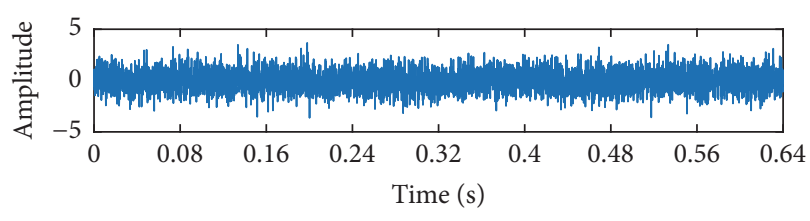

(c) The Gauss noise

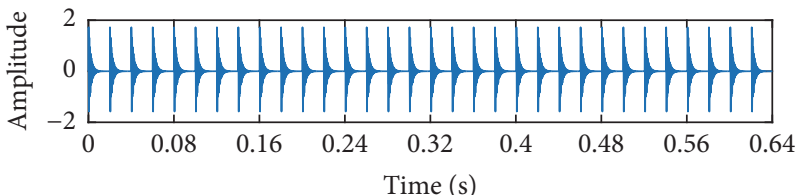

(b) Outer ring fault simulation signal

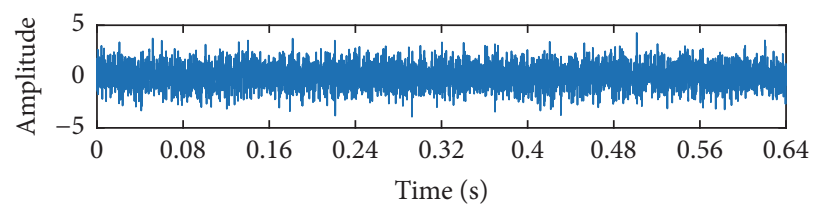

(d) The compound signal

FIgURE 3: Time domain waveform of simulation signal.

where $y_{0}$ is the possible amplitude modulator; $f_{n}$ is the natural frequency; $t_{0}$ is a single cycle sampling time; $g$ is the damping factor. The parameters of the outer ring simulation signal are shown in Table 2.

The time domain waveform of the inner ring and outer ring faulty simulation signals is shown in Figures 3(a) and $3(b)$; Figure 3(c) is the Gauss noise; Figure $3(d)$ is the compound fault simulation signal. It can be seen from Figure 3(d) that the inner and outer shock characteristics are submerged by noise. The compound fault simulation signal is demodulated by envelope (Figure 4), and the characteristic frequency and its frequency doubling of the outer ring fault can be seen from the envelope spectrum. However the fault characteristics of inner ring are submerged by the noise frequency, so the envelope spectrum can not accurately reflect the characteristics of fault impact.

Figure 5 shows the fast spectral kurtosis analysis on the compound fault simulation signals. The resonance frequency band of the outer ring can be recognized while the resonance frequency of the inner ring fault is failed to recognize. Figure 6 is the envelope spectrum after the FSK filtering. As can be seen from Figure 6, the characteristic frequency and frequency doubling of the outer race fault are successfully extracted, and the characteristic frequency of the inner ring fault can not be extracted. This shows that the FSK algorithm is prone to cause misdiagnosis or missed diagnosis when dealing with compound fault.

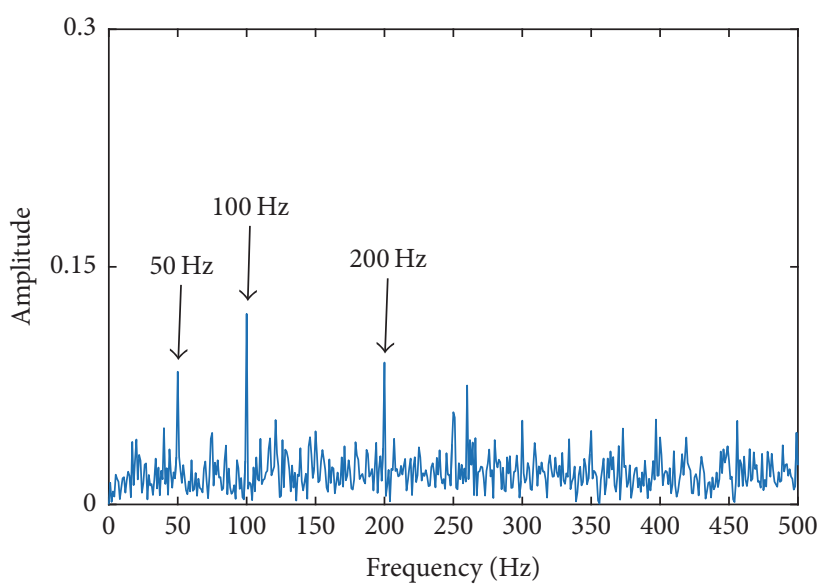

FIGURE 4: The envelope spectrum of simulation signal.

The method of maximum correlation kurtosis deconvolution (MCKD) is used to avoid the misjudgment and missed diagnosis of compound fault. The MCKD method has two important parameters that need to be set. One is the deconvolution cycle $(T)$, and the other is the filter length $(L)$. The filter length $(L)$ is in the range of 10 to 300 , and the optimal solution is obtained at 10 step sizes. Finally, the length of the outer ring fault deconvolution filter is set to 50 , and 
TABLE 3: Parameters of rolling bearing.

\begin{tabular}{lccccc}
\hline Inside diameter $/ \mathrm{mm}$ & Outside diameter $/ \mathrm{mm}$ & Ball diameter $/ \mathrm{mm}$ & Pitch diameter $/ \mathrm{mm}$ & Number of balls $/$ 个 & Contact angle $/\left(^{\circ}\right)$ \\
\hline 17 & 40 & 6.7 & 28.5 & 8 & 0 \\
\hline
\end{tabular}



FIGURE 5: The spectral kurtosis of simulation signal.

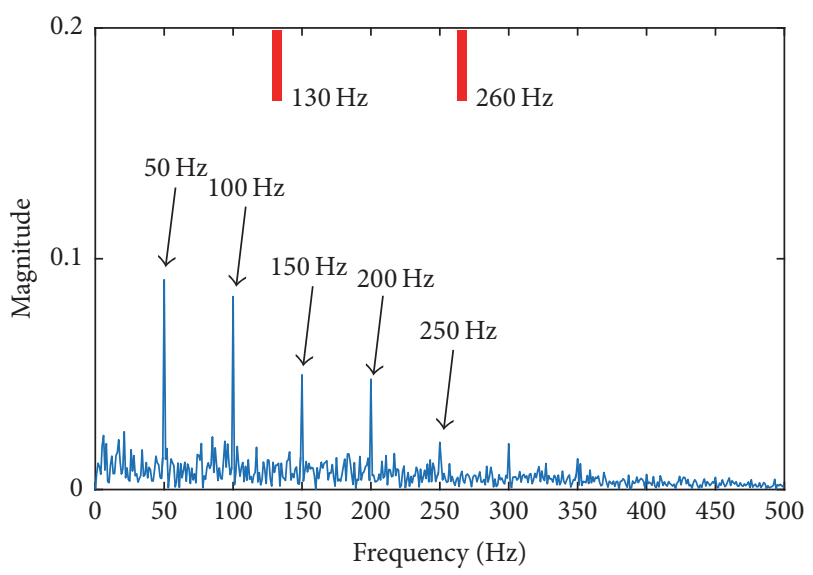

FIGURE 6: The envelope spectrum after FSK filtering.

the length of inner ring fault deconvolution filter is set to 200. The deconvolution period of the inner and outer rings is calculated by the formula as follows.

$$
\begin{aligned}
& T_{o}=\frac{f_{s}}{f_{o}}=\frac{8192}{50}=163.8, \\
& T_{i}=\frac{f_{s}}{f_{i}}=\frac{8192}{130}=63 .
\end{aligned}
$$

The outer and inner ring fault features after the deconvolution operation are shown in Figures 7(a) and 7(b). It can be seen from the time domain graph that the original shock characteristics can be reduced to a certain extent.

Figure $8(\mathrm{a})$ is the FSK analysis of the signal after the deconvolution filtering. The resonance band of the outer
TABLE 4: Fault feature frequency of rolling bearing.

\begin{tabular}{lccc}
\hline Types of failures & Outer ring & Inner ring & Rolling element \\
\hline Defect frequencies $/ \mathrm{Hz}$ & 88 & 143 & 115 \\
\hline
\end{tabular}

race fault is found. Bandpass filtering is performed on this frequency band, and then the envelope spectrum is analyzed. In the envelope spectrum, the characteristic frequency of the outer race of the bearing is found. Similarly, the inner ring fault signal is analyzed in the same way. The inner ring fault resonance band is found in the FSK method and the characteristic frequency of the inner ring fault is found in the envelope spectrum (Figure 9).

\section{Experimental Analysis}

The effectiveness of the proposed method is verified by the experimental data of rolling bearings at Case Western Reserve University in the United States [16]. The experimental equipment is shown in Figure 10. The bearing model is JEMSKF6023-2RS. The most minor dimensions of 0.007inch fault data are analyzed. The sampling frequency is $12 \mathrm{kHz}$, and the rotation speed of the shaft is $1730 \mathrm{r} / \mathrm{min}$. The bearing parameter dimensions are shown in Table 3, and the frequency of each fault characteristic of the bearing is shown in Table 4 . The time domain waveform of 0.007 -inch fault data is shown in Figure 11.

The MCKD is adopted to separate the fault features by reasonably selecting the filter length $L$ and the convolution cycle $T$. Among them, the filter length is within the range of $[10,300]$, and 10 is chosen as the step size. Finally, the parameter of MCKD is determined, the filter length of the rolling element is 230 , and the filter length of the inner ring is 200 . The deconvolution period is the ratio of the sampling frequency to the characteristic frequency of the fault, which can be calculated by the following formula:

$$
\begin{aligned}
T_{\text {ball }} & =\frac{f_{s}}{f_{\text {ball }}}=\frac{12000}{115}=104.3 \\
T_{i} & =\frac{f_{s}}{f_{i}}=\frac{12000}{143}=83.9 .
\end{aligned}
$$

Figure 12(a) is the time domain waveform of the rolling element fault after MCKD. Figure 12(b) is the time domain waveform of the inner ring fault after MCKD. From the time domain waveform, it can be seen that the fault characteristics are revealed by the MCKD.

The FSK of the rolling element and inner ring fault after deconvolution is carried out, and the spectral kurtosis resonance band can be clearly reflected in the spectral kurtosis. 


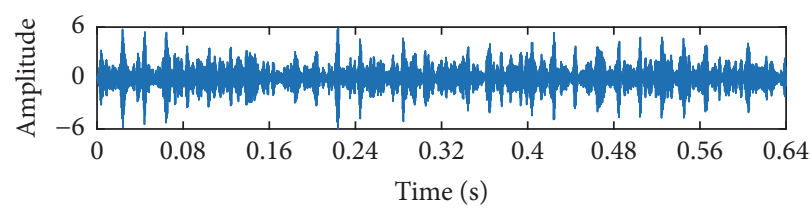

(a) The time domain waveform of outer ring fault

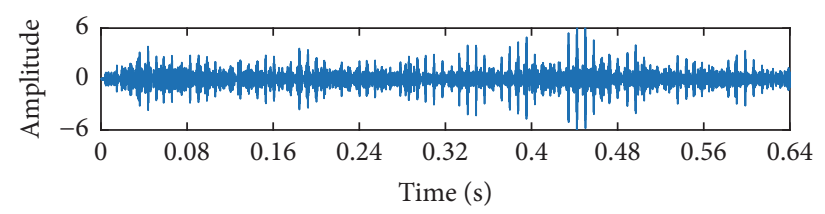

(b) The time domain waveform of inner ring fault

FIGURE 7: The time domain waveform after MCKD.

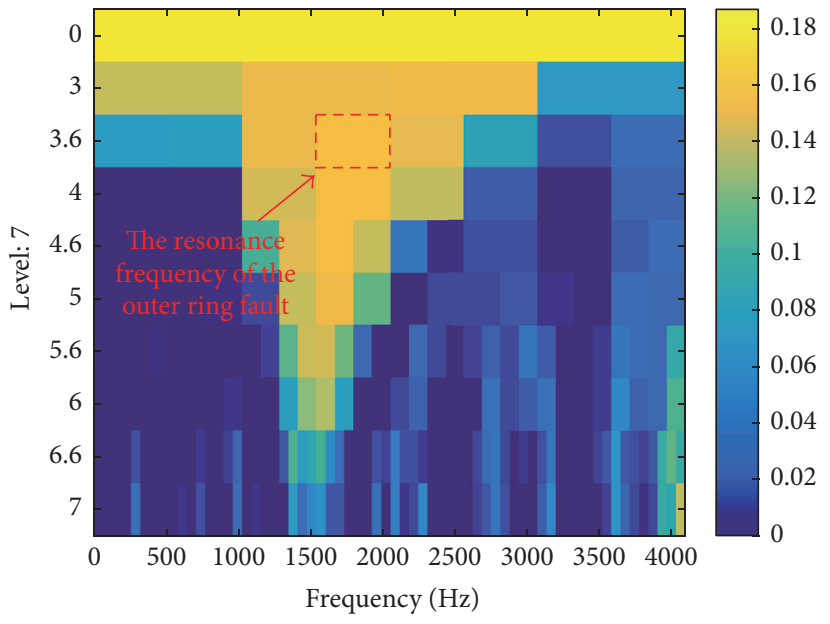

(a) The spectral kurtosis of outer ring fault

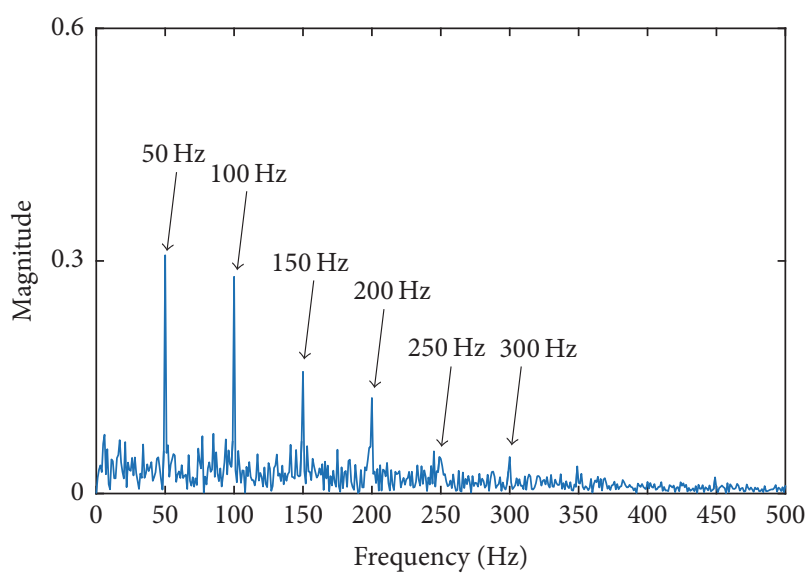

(b) The envelope spectrum after FSK filtering

FIGURE 8: The fast spectral kurtosis analysis of the outer ring fault.

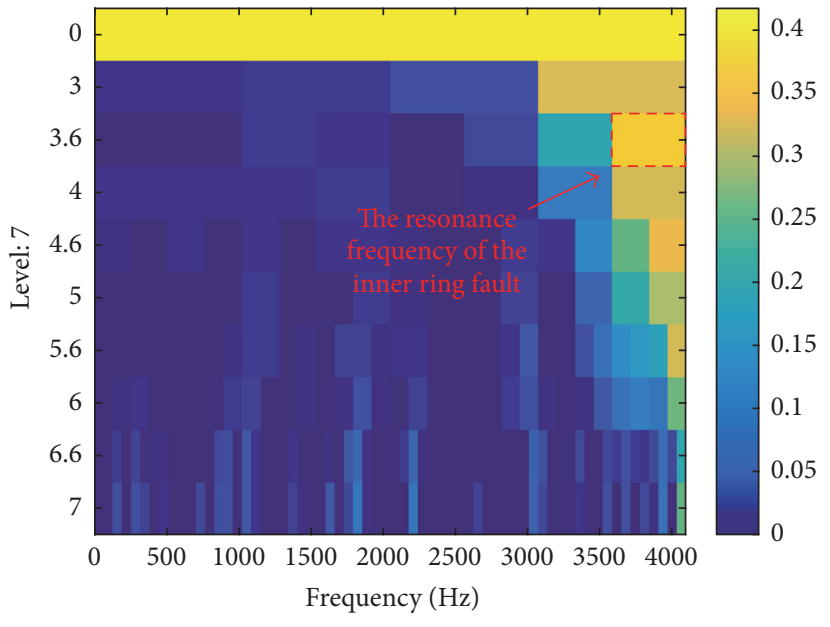

(a) The spectral kurtosis of inner ring fault

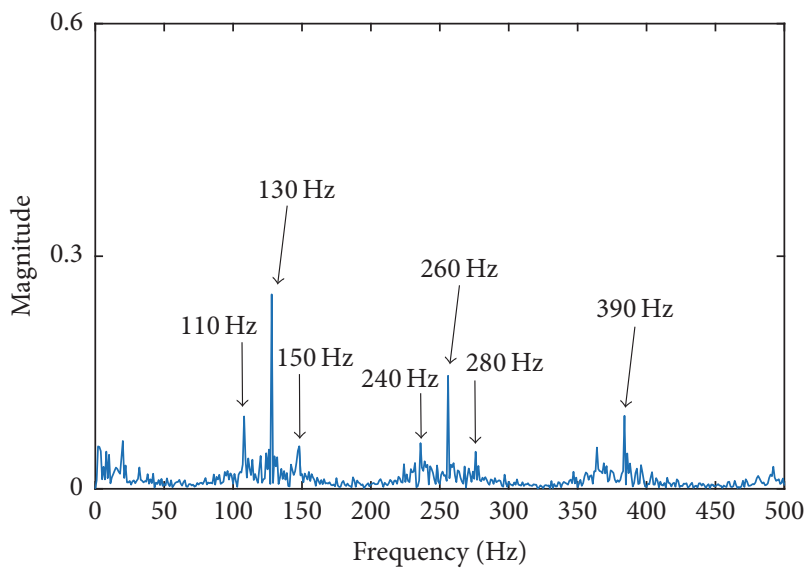

(b) The envelope spectrum after FSK filtering

FIGURE 9: The fast spectral kurtosis analysis of the inner ring fault.

Figure 13(a) reflects the resonance band determined by the FSK of the inner ring fault and Figure 13(b) is the envelope spectrum of the filtered signal in the previous resonance band. Figure 13(c) reflects the resonance band determined by the FSK of the rolling element fault and Figure 13(d) is the envelope spectrum of the filtered signal in the previous resonance band.

Figure 14 is the FSK of the original compound fault signal. From the spectral kurtosis diagram, it can be seen that the determined rolling element fault frequency band is 


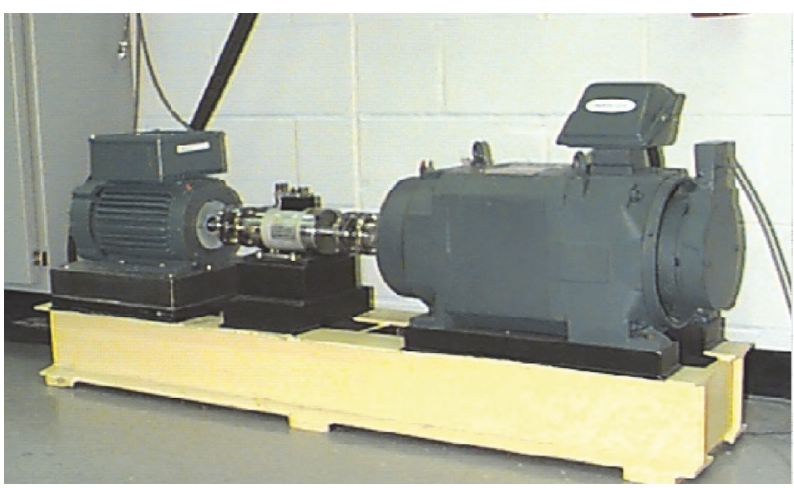

FIGURE 10: The bearing test stand of Case University.

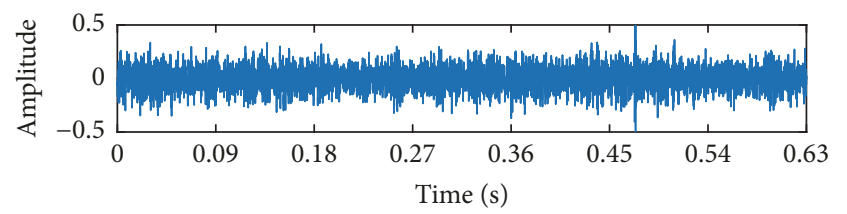

(a) The time domain waveform of rolling element fault

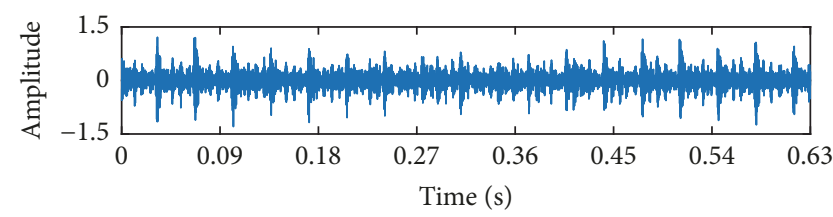

(b) The time domain waveform of inner ring fault

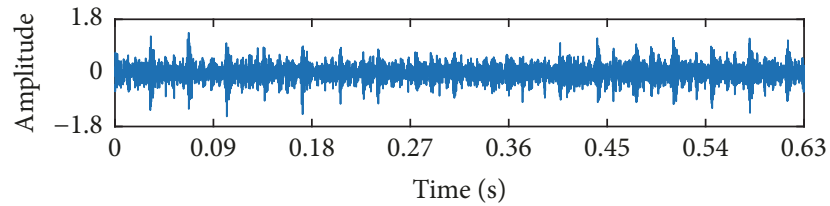

(c) The time domain waveform of the compound signal

FIGURE 11: The time domain waveform of 0.007-inch fault data.

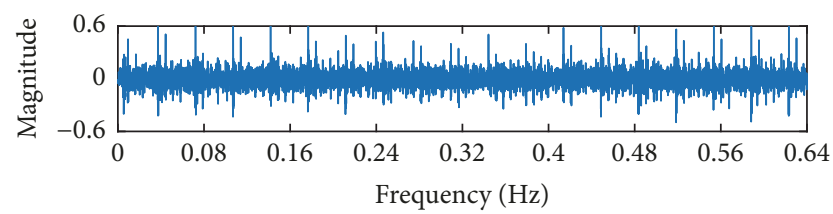

(a) The time domain waveform of rolling element fault

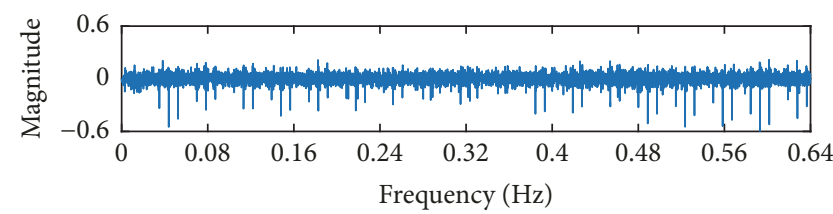

(b) The time domain waveform of inner ring fault

FIGURE 12: The time domain waveform after MCKD.

inaccurate, while the inner ring fault band is missing. That is to say, the direct application of FSK to deal with compound fault signals results in missed diagnosis.

\section{Experimental Analysis}

In order to further verify the effectiveness of the proposed method, the simulation experiment of compound fault of inner and outer race of rolling bearing was completed in the simulation experiment platform of QPZZ bearing fault. The bearing model is $6205 \mathrm{E}$. The grooves which are $1.5 \mathrm{~mm}$ deep and $0.2 \mathrm{~mm}$ wide were processed in the inner ring and the outer ring to simulate the bearing compound failure. The vibration signal is collected by the acceleration sensor mounted on the bearing seat. The sampling frequency is
$12800 \mathrm{~Hz}$, and the motor speed is $1466 \mathrm{r} / \mathrm{min}$. Figure 15 is the structural diagram of the test platform. Figure 16 is the compound fault bearing. The bearing parameters are shown in Table 5.

The characteristic frequency of bearing inner and outer ring fault is calculated as

$$
\begin{aligned}
& f_{i}=\frac{Z}{2}\left(1+\frac{d}{D} \cos \alpha\right) \frac{N}{60}=132.2 \mathrm{~Hz} \\
& f_{\mathrm{o}}=\frac{Z}{2}\left(1-\frac{d}{D} \cos \alpha\right) \frac{N}{60}=87.7 \mathrm{~Hz} .
\end{aligned}
$$

The 8192 data points of the collected vibration signals were selected for analysis and the time domain waveform of the compound fault is shown in Figure 17. Figure 18(a) is a 


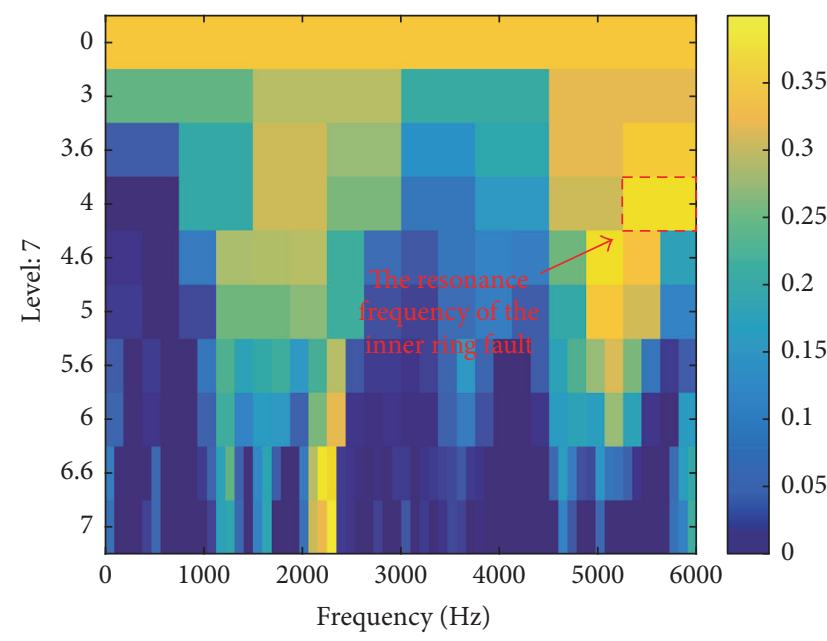

(a) The spectral kurtosis of inner ring fault

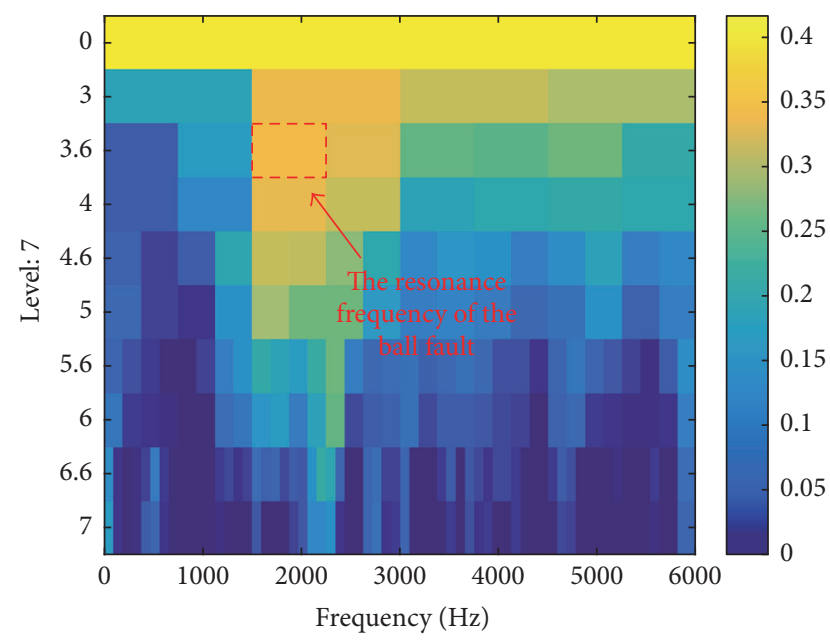

(c) The spectral kurtosis of rolling element fault



(b) The envelope spectrum after FSK filtering

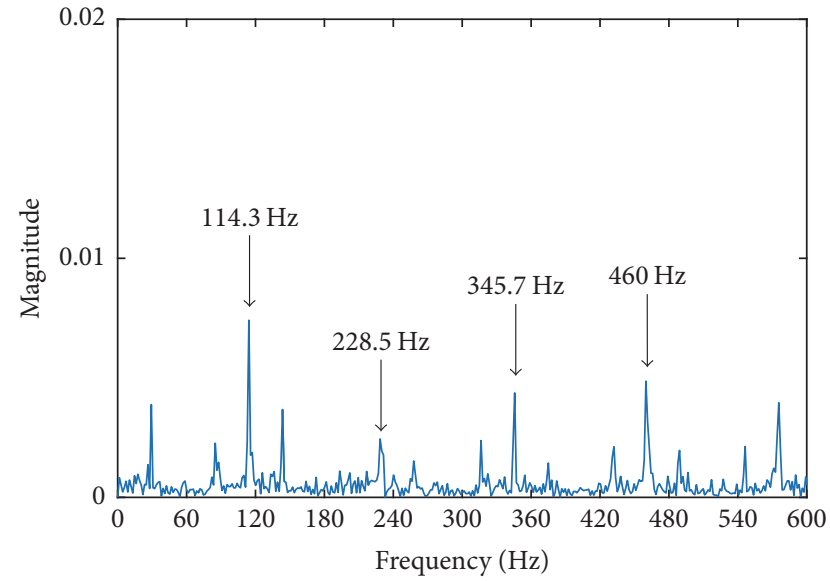

(d) The envelope spectrum after FSK filtering

FIGURE 13: The FSK analysis of signals after MCKD.

TABLE 5: Parameters of rolling bearing.

\begin{tabular}{lccccc}
\hline Inside diameter $/ \mathrm{mm}$ & Outside diameter $/ \mathrm{mm}$ & Ball diameter $/ \mathrm{mm}$ & Pitch diameter $/ \mathrm{mm}$ & Number of balls & Contact angle $/\left({ }^{\circ}\right)$ \\
\hline 25 & 52 & 7.9 & 39 & 9 & 0
\end{tabular}

direct analysis of the original compound fault signal by FSK. Only the approximate outer ring resonant frequency band can be found from the spectral kurtosis. Figure 18(b) is the envelope spectrum of the signal after filtering by the spectral kurtosis. In the envelope spectrum, only the characteristic frequency of the outer ring fault can be extracted, and the characteristic frequency of the inner ring fault can not be extracted.

The MCKD method is used as the preprocessing of the FSK. First, the parameters of MCKD are reasonably selected. Among them, the filter length is within the range of $[10,300]$, and 10 is chosen as the step size. Finally, the parameter of MCKD is determined, the filter length of the outer ring is 120 , and the filter length of the inner ring is 200 . The deconvolution period is the ratio of the sampling frequency to the characteristic frequency of the fault, which can be calculated by the following formula:

$$
\begin{aligned}
T_{\text {ball }} & =\frac{f_{s}}{f_{\text {ball }}}=\frac{12800}{87.7}=145.95 \\
T_{i} & =\frac{f_{s}}{f_{i}}=\frac{12800}{132.2}=96.82 .
\end{aligned}
$$

Figure 19(a) is the time domain waveform of the outer ring fault after MCKD. Figure 19 (b) is the time domain waveform of the inner ring fault after MCKD. From the time domain waveform, it can be seen that the fault characteristics are revealed to a certain extent by the MCKD.

The FSK analysis of the rolling element and inner ring fault after deconvolution is carried out, and the spectral 


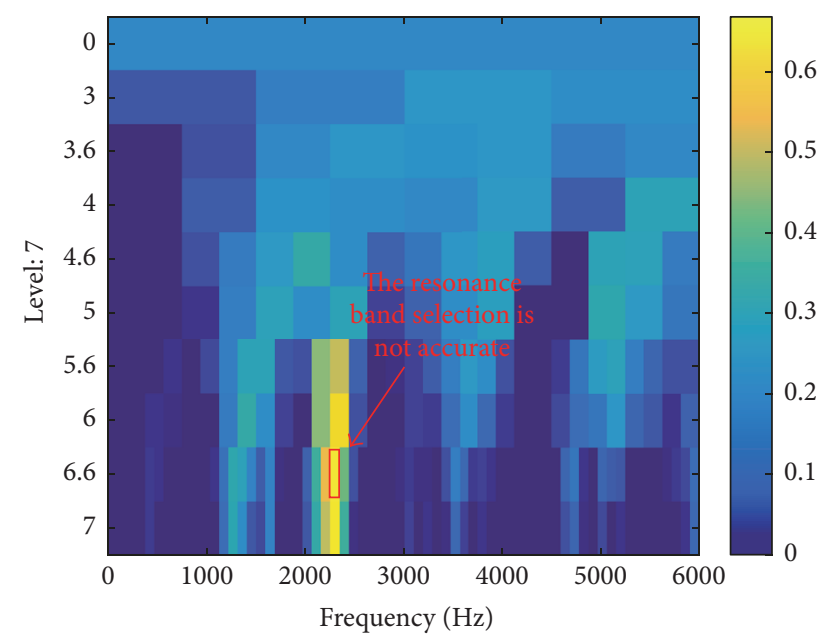

FIGURE 14: The fast spectral kurtosis analysis of original compound fault signal.

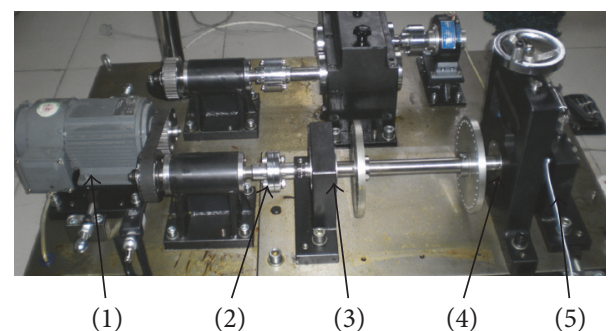

FIGURE 15: The QPZZ experiment platform. (1) The drive motor, (2) the coupling, (3) the bearing housing at normal bearing seat, (4) the loading device, and (5) the bearing housing at fail bearing seat.

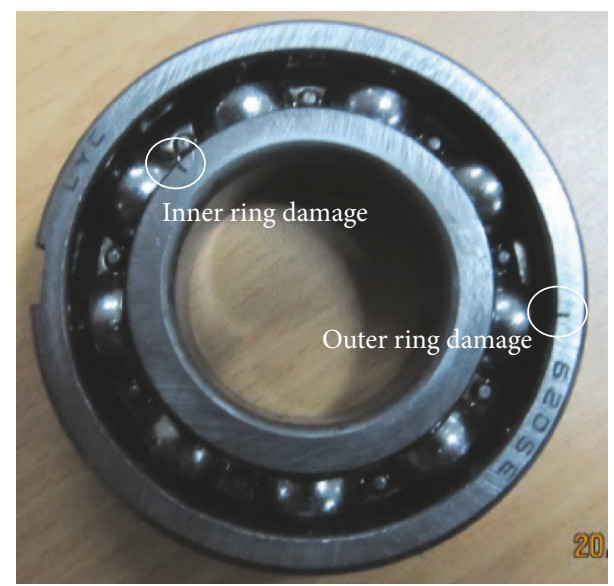

FIGURE 16: Bearing outer and inner ring compound fault.

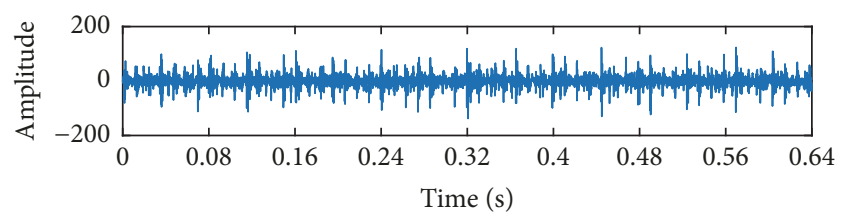

FIgURE 17: The time domain waveform of the compound fault.

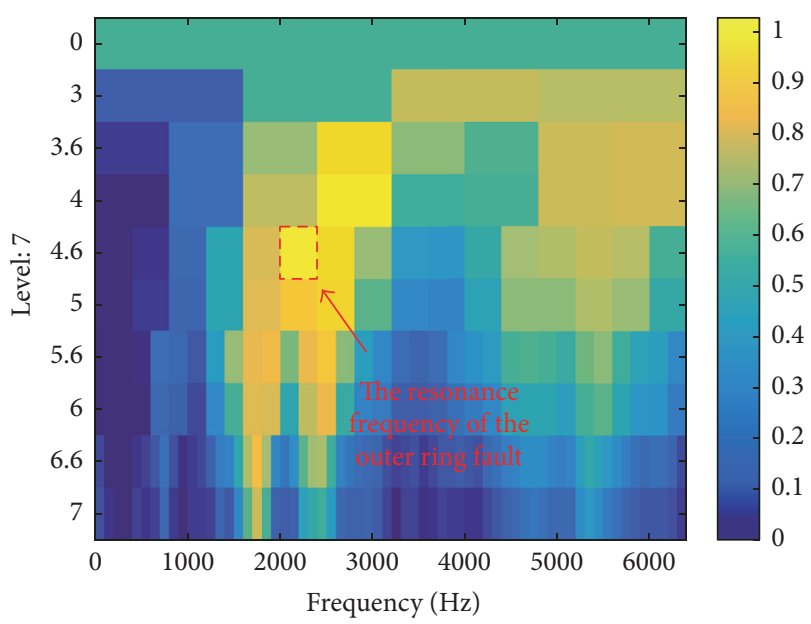

(a) The spectral kurtosis of the compound fault

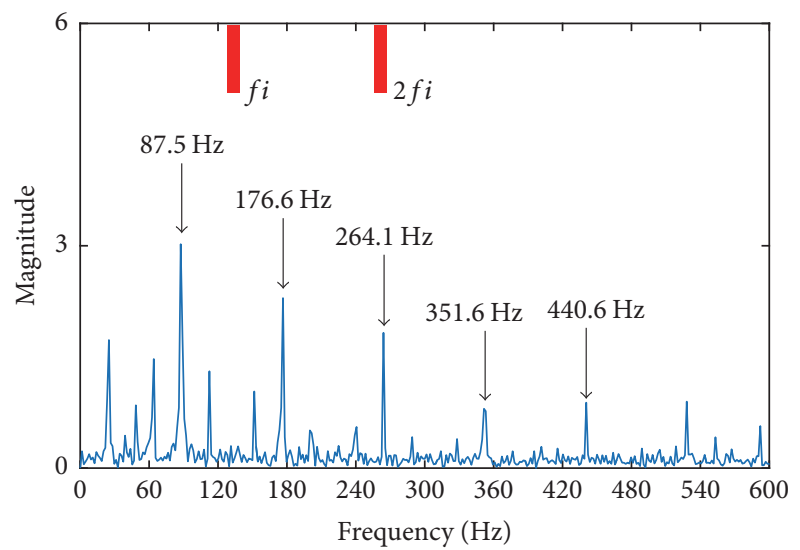

(b) The envelope spectrum after FSK filtering

FIGURE 18: The fast spectral kurtosis analysis of original compound fault signal.

kurtosis resonance band can be clearly reflected in the spectral kurtosis. Figure 20(a) reflects the resonance band determined by the FSK of the outer ring fault and Figure 20(b) is the envelope spectrum of the filtered signal in the previous resonance band. Figure 20(c) reflects the resonance band determined by the FSK of the inner ring fault and Figure 20(d) is the envelope spectrum of the filtered signal in the previous resonance band.

As shown in Figure 20, this method decomposes the fault feature information of the compound fault signal in different resonance frequency bands into different signal components. The resonance frequency band of the bearing inner and outer ring fault is identified accurately by spectral kurtosis. After filtering and envelope operation, the fault feature information contained in the resonance frequency band is clearly extracted.

\section{Conclusions}

Fast spectral kurtosis is one of the most classical methods in bearing fault diagnosis. However, because of the limitation 


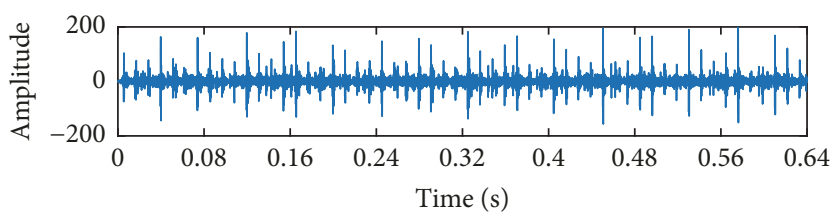

(a)

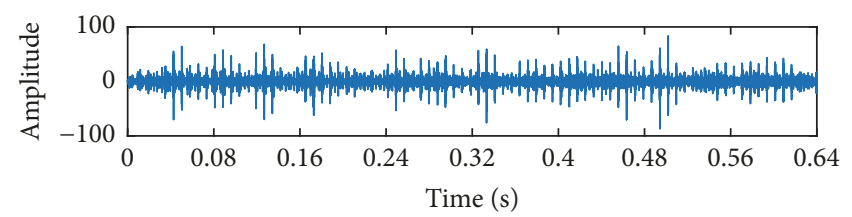

(b)

FIGURE 19: The time domain waveform after MCKD.

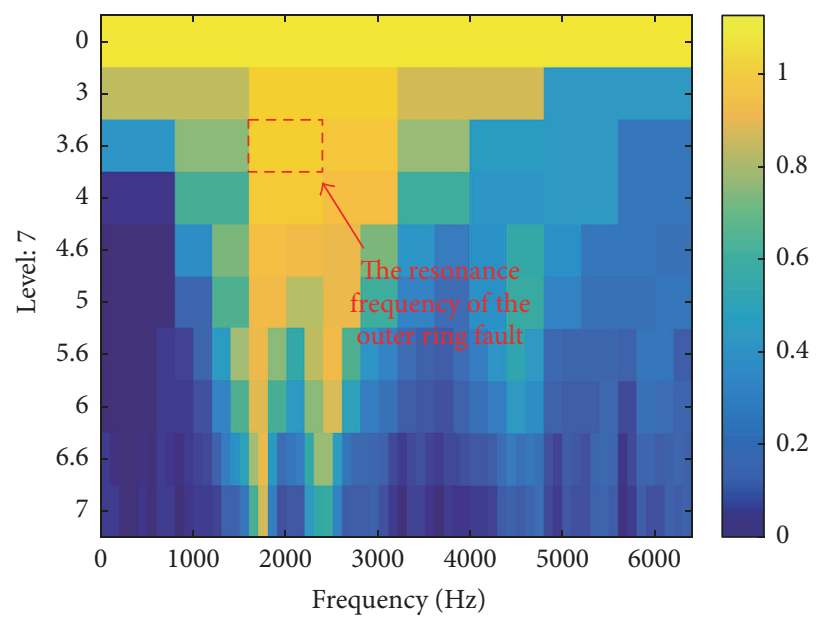

(a) The spectral kurtosis of outer ring fault

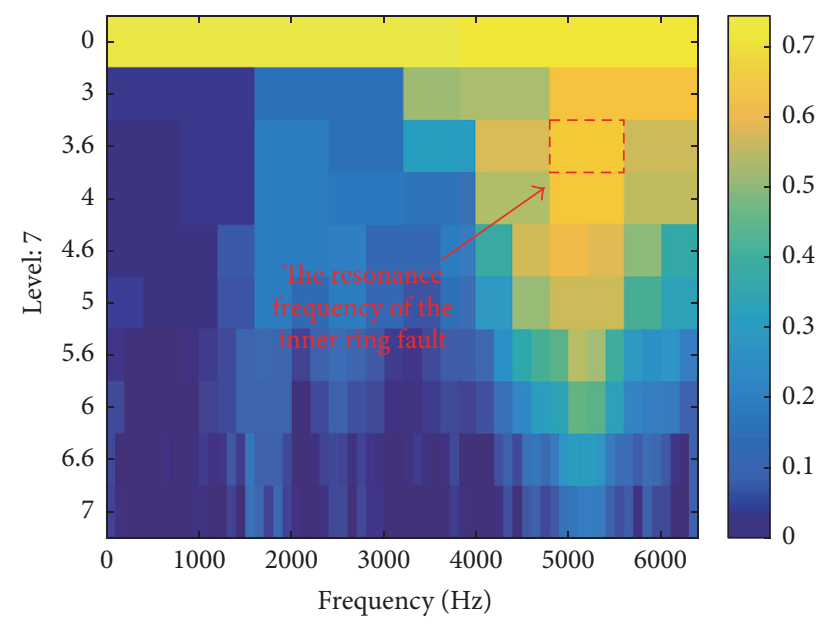

(c) The spectral kurtosis of inner ring fault

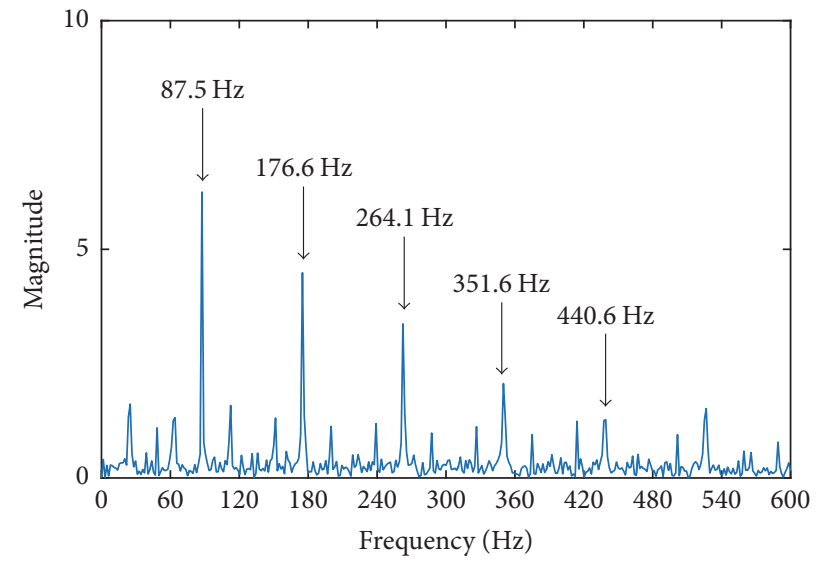

(b) The envelope spectrum after FSK filtering

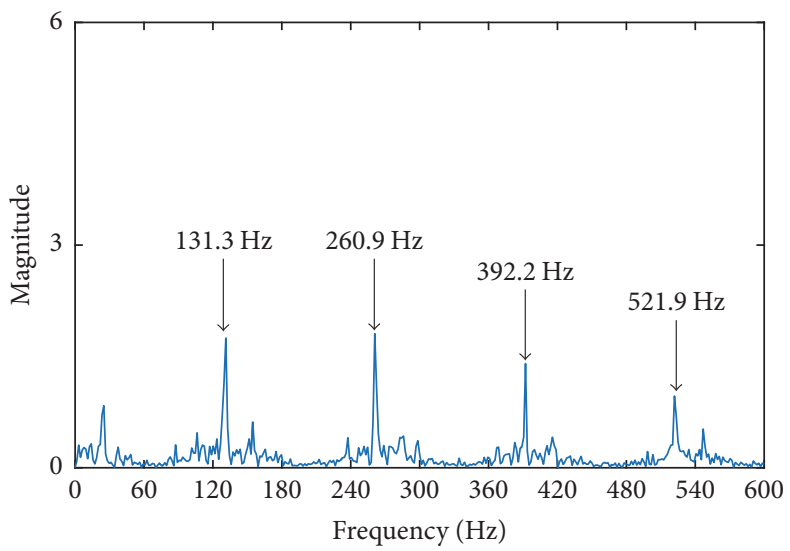

(d) The envelope spectrum after FSK filtering

FIGURE 20: The fast spectral kurtosis analysis of signals after MCKD.

of its own algorithm, it is not possible to accurately extract multiple fault information located in different resonance frequency bands. In view of the limitation of FSK in dealing with compound fault of rolling bearings, a method is proposed in this paper to assist FSK to select the resonance band of the compound fault by using MCKD as the preprocess. Firstly, the parameter of MCKD is selected reasonably, and then the original signal is filtered to separate the compound fault information at different resonance frequency bands by
MCKD. Then the filtered signals after MCKD are analyzed by FSK. Simulation and experimental analysis show that the proposed method can effectively extract compound fault in different resonance frequency bands. The method proposed in this paper solves the limitation of fast spectral kurtosis in bearing compound fault of bearing. In the future, we will work in the multicomponent compound fault of gear and bearing in the gearbox transmission chain and further correct the algorithm so that the application range is expanded. 


\section{Conflicts of Interest}

The authors declare no conflicts of interest related to this manuscript.

\section{Authors' Contributions}

All authors have approved the manuscript.

\section{Acknowledgments}

This work was supported by the Fundamental Research Funds for the Central Universities (no. 2017XS133 and no. 2018QN093) and the National Natural Science Foundation of China (no. 51777075).

\section{References}

[1] P. A. Delgado-Arredondo, D. Morinigo-Sotelo, R. A. OsornioRios, J. G. Avina-Cervantes, H. Rostro-Gonzalez, and R. D. J. Romero-Troncoso, "Methodology for fault detection in induction motors via sound and vibration signals," Mechanical Systems and Signal Processing, vol. 83, pp. 568-589, 2017.

[2] A. Glowacz, W. Glowacz, Z. Glowacz, and J. Kozik, "Early fault diagnosis of bearing and stator faults of the single-phase induction motor using acoustic signals," Measurement, vol. 113, pp. 1-9, 2018.

[3] X. An and L. Pan, "Bearing fault diagnosis of a wind turbine based on variational mode decomposition and permutation entropy," Proceedings of the Institution of Mechanical Engineers, Part O: Journal of Risk and Reliability, vol. 231, no. 2, pp. 200206, 2017.

[4] H. Zhao, M. Sun, W. Deng, and X. Yang, "A new feature extraction method based on EEMD and multi-scale fuzzy entropy for motor bearing," Entropy, vol. 19, no. 1, article 14, 2017.

[5] Y. Gao, F. Villecco, M. Li, and W. Song, "Multi-scale Permutation entropy based on improved LMD and HMM for rolling bearing diagnosis," Entropy, vol. 19, no. 4, article 176, 2017.

[6] D. Wang, W. Guo, and X. Wang, "A joint sparse wavelet coefficient extraction and adaptive noise reduction method in recovery of weak bearing fault features from a multi-component signal mixture," Applied Soft Computing, vol. 13, no. 10, pp. 4097-4104, 2013.

[7] D. Zhao, J. Li, W. Cheng, and W. Wen, "Compound faults detection of rolling element bearing based on the generalized demodulation algorithm under time-varying rotational speed," Journal of Sound and Vibration, vol. 378, pp. 109-123, 2016.

[8] M. Yu, Z. Feng, J. Huang, and Y. Yu, "Characteristic extraction of rolling bearing compound faults of aero-engine," Journal of Vibroengineering, vol. 19, no. 6, pp. 4285-4299, 2017.

[9] F. Hemmati, W. Orfali, and M. S. Gadala, "Roller bearing acoustic signature extraction by wavelet packet transform, applications in fault detection and size estimation," Applied Acoustics, vol. 104, pp. 101-118, 2016.

[10] C. Zhang, Z. Li, C. Hu, S. Chen, J. Wang, and X. Zhang, "An optimized ensemble local mean decomposition method for fault detection of mechanical components," Measurement Science and Technology, vol. 28, no. 3, Article ID 035102, 2017.

[11] J. Antoni and R. B. Randall, "The spectral kurtosis: application to the vibratory surveillance and diagnostics of rotating machines," Mechanical Systems and Signal Processing, vol. 20, no. 2, pp. 308-331, 2006.

[12] N. Sawalhi, R. B. Randall, and H. Endo, "The enhancement of fault detection and diagnosis in rolling element bearings using minimum entropy deconvolution combined with spectral kurtosis," Mechanical Systems and Signal Processing, vol. 21, no. 6, pp. 2616-2633, 2007.

[13] H. Y. Liu, W. G. Huang, S. B. Wang, and Z. K. Zhu, "Adaptive spectral kurtosis filtering based on Morlet wavelet and its application for signal transients detection," Signal Processing, vol. 96, pp. 118-124, 2014.

[14] G. L. McDonald, Q. Zhao, and M. J. Zuo, "Maximum correlated Kurtosis deconvolution and application on gear tooth chip fault detection," Mechanical Systems and Signal Processing, vol. 33, pp. 237-255, 2012.

[15] H. Wang, J. Chen, and G. Dong, "Fault diagnosis method for rolling bearing's weak fault based on minimum entropy deconvolution and sparse decomposition," Journal of Mechanical Engineering, vol. 49, no. 1, pp. 88-94, 2013.

[16] Bearing Data Center-Case Western Reserve University, Cleveland, Ohio, USA, http://www.eecs.case.edu/laboratory/bearing. 


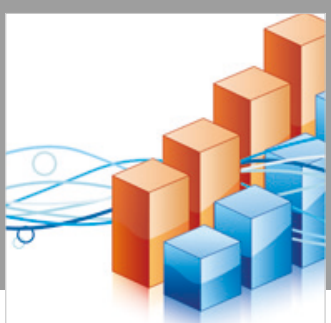

Advances in

Operations Research

\section{-n-m}
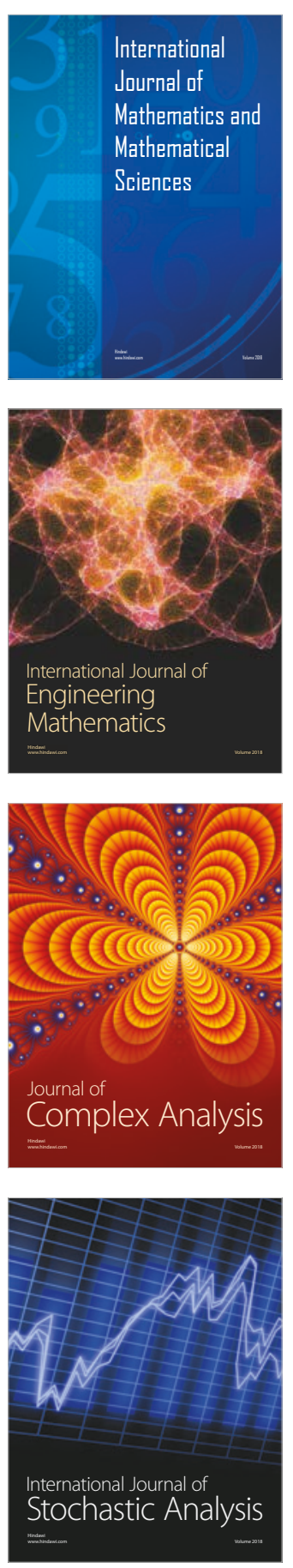
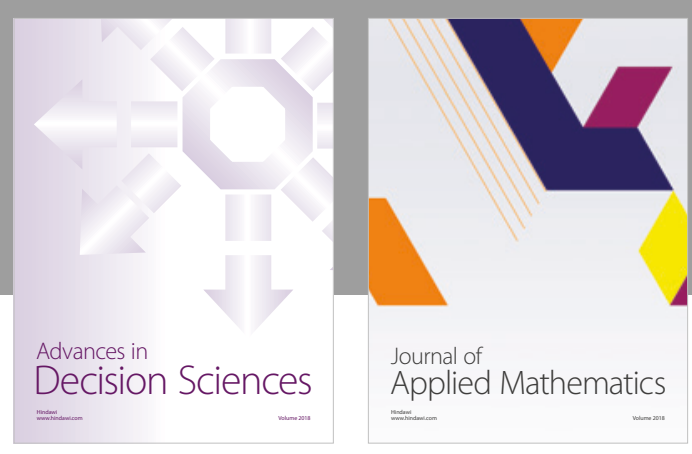

Journal of

Applied Mathematics
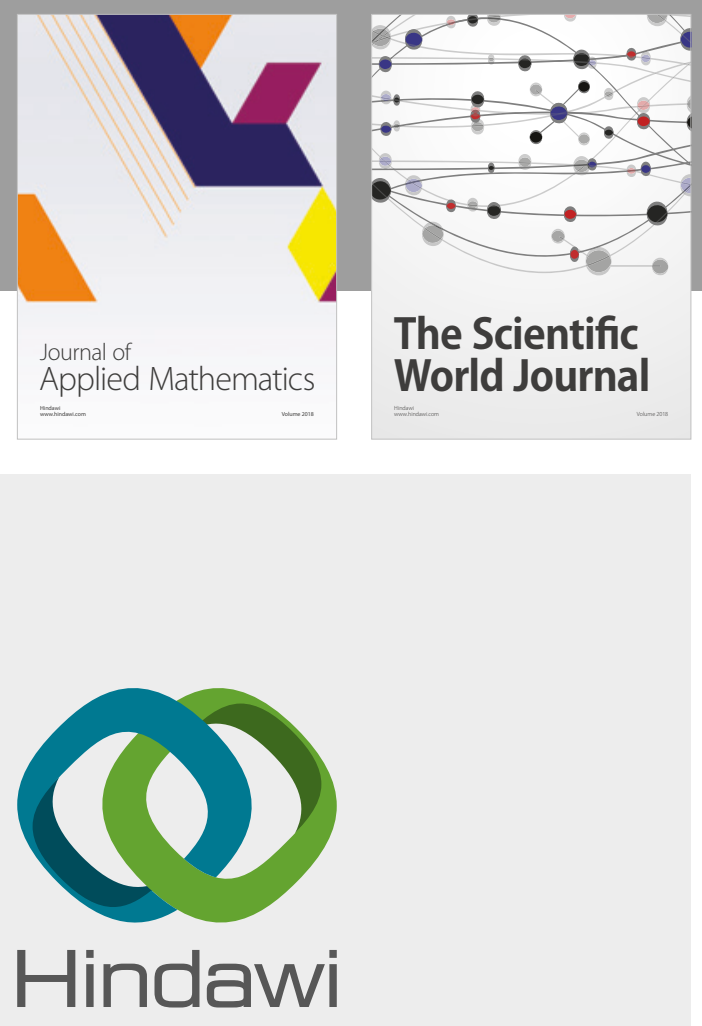

Submit your manuscripts at

www.hindawi.com

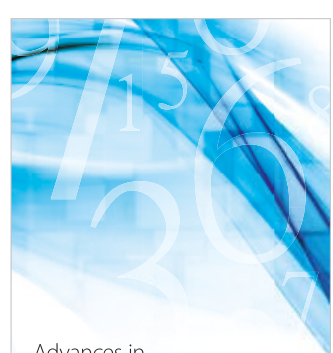

Advances in
Numerical Analysis
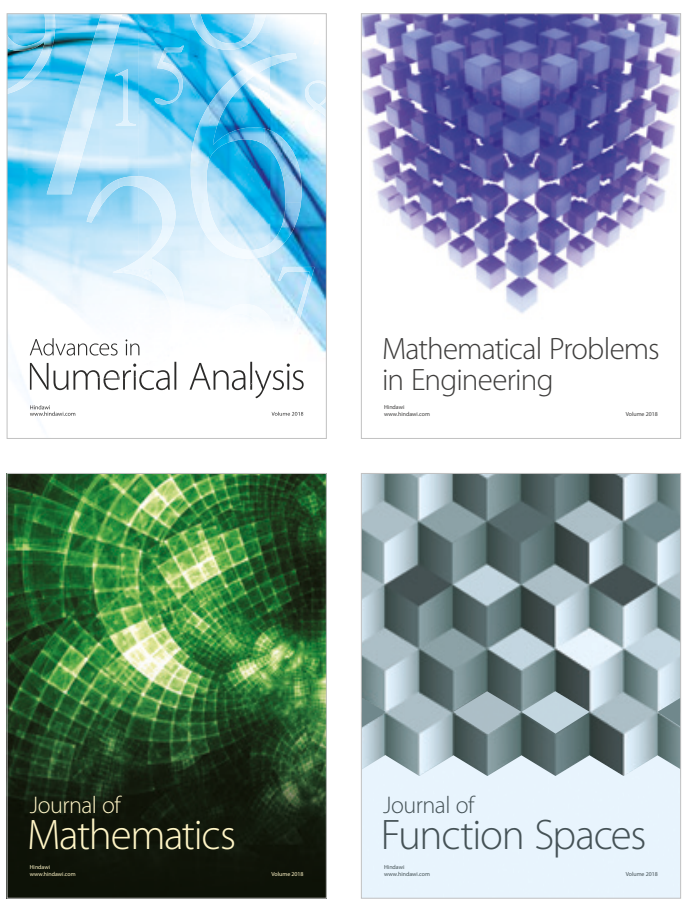

Mathematical Problems in Engineering

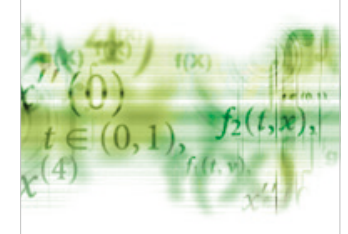

International Journal of

Differential Equations

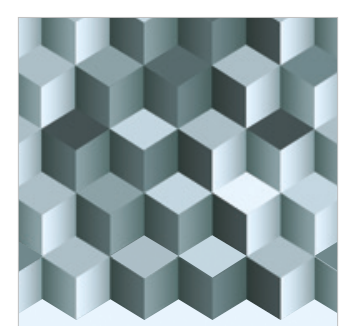

Journal of

Function Spaces

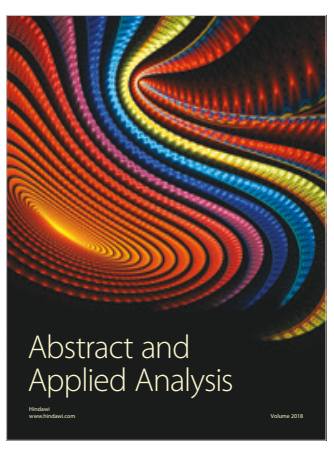

The Scientific

World Journal

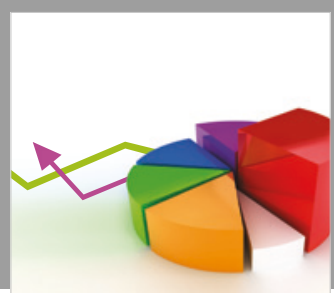

Journal of

Probability and Statistics
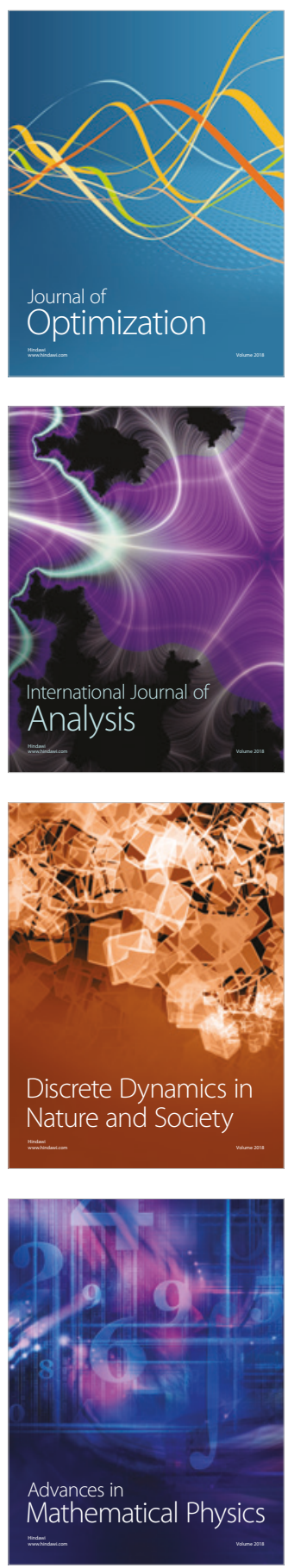